\section{Possible effect of medically administered antibiotics on the mutans streptococci: implications for reduction in decay}

Loesche WJ, Eklund SA, Mehlisch DF, Burt B. Possible effect of medically administered antibiotics on the mutans streptococci: implications for reduction in decay. Oral Microbiol Immunol 1989: 4: 77-81.

The decline in dental caries in children in North America, Scandinavia, Britain, Ireland and many Commonwealth nations is well documented. The multiple uses of fluoride can account for most, but not all, of this reduction. In this investigation, data are provided which suggest a relationship between antibiotic usage for medical purposes and a decline in both mutans streptococci (MS) and caries. Children attending Grades 1 and 2 in the Coldwater, Michigan school system and who reportedly never received antibiotics had significantly higher proportions of MS in the fissure plaques of first molars than subjects who received antibiotics. The level of decay in the primary dentition was inversely related to the reported usage of antibiotics. The frequent usage of antibiotics could reduce the incidence of dental caries by delaying the colonization of the teeth by the MS. This was evaluated by a prospective study in infants to determine what effect reported antibiotic usage would have on the colonization of newly erupting primary teeth. Only 2 of 10 infants cultured at 2 to 3 week intervals for periods up to 1 year after tooth eruption became colonized by the MS. One had never received antibiotics and the second had been on antibiotics for a single 5-day period. Seven of the 8 non-colonized infants had received antibiotic therapy for periods ranging from 10 to 181 days. Both the Coldwater study and the prospective study of infants suggested a relation between frequency of antibiotic usage for medical purposes and the MS levels on the teeth.
Walter J. Loesche ${ }^{1}$, Stephen A. Eklund ${ }^{2}$, David F. Mehlisch ${ }^{3}$, Brian Burt ${ }^{2}$

${ }^{1}$ Department of Biologic and Materials Sciences, ${ }^{2}$ Program in Dental Public Health, University of Michigan, Ann Arbor, ${ }^{3}$ Department of Community Dentistry and Human Behavior, Southern Illinois University, U.S.A.
Key words: antibiotics; mutans streptococci.

W. J. Loesche University of Michigan School of Dentistry, Ann Arbor, MI48109, USA.

Accepted for publication September 8, 1988
The decline in dental caries in children residing in North America, Scandinavia, Britain, Ireland and the various Commonwealth nations is well documented (10). Evidence points to water fluoridation and fluoridated dentifrices as the major factors responsible for this reduction, but fluoride may not account for all the decline (3). The reduction has occurred in countries like Sweden where there is no water fluoridation (18), and in non-fluoridated areas in the U.S. (6). Indeed, a reduction of decay in the primary dentition is being observed in Japan (Matsukudo, pers. comm.) where there is neither water fluoridation nor widespread usage of fluoride dentifrices. What then besides fluorides could be contributing to this decrease in dental caries? There is no reason to suspect that oral hygiene in the absence of fluoridated dentifrices has played a role, as even the best oral hygiene habits have little effect on the incidence of decay $(2,13,15)$. There is no evidence that a decreased consumption of sweetened foods or a decreased frequency of snacking has contributed to this decline (7) and a genetic change in the host in untenable.

Since Keyes (16) demonstrated the transmissible nature of dental decay in animal models, numerous investigators have provided evidence that the mutans streptococci (MS), of which Streptococcus mutans is the predominant human type, are statistically associated with human dental decay (21). If the MS are human cariogens, then the decline in decay could reflect a decrease in their levels, or a delay in the timing of an MS infection in children (22). The salivary levels of the MS and lactobacilli (also implicated in human decay) had de- creased significantly in 9 to 12 year old Swedish school children between 1972 and 1984, and this decrease was coincident with a reduction in the mean decayed and filled surface scores from 12.8 to 3.0 (17). Likewise, the salivary levels of U.S. children attending a pediatric dental clinic had decreased by about $90 \%$ between 1973-74 and 1981-82 (22).

Fluoridation does not seem to lower MS levels $(4,30)$. However, antibiotics do temporarily reduce the salivary MS levels (25), and during the time span that decay has been declining the medical usage of antibiotics, has increased substantially (3). This raises the possibility that some part of the decline in both MS and in decay could be secondary to the medical usage of antibiotics $(3,22)$. The possiblity that antibiotic usage could reduce decay has been sug- 
gested by studies of caries prevalence in children with rheumatic fever $(14,20)$ and by clinical observations (5).

There has been a marked decline in mortality due to rheumatic fever in the U.S. (12, 26). A retrospective study has shown that while the decline in occurrence of rheumatic fever antedated the availability of antibiotics, the rates of decline in national mortality due to rheumatic carditis accelerated fourfold with the advent of antibiotics (26). As the Group A streptocci that are implicated in rheumatic fever and the MS are sensitive to the same antibiotics, this data on rheumatic fever is supportive of the hypothesis that frequent antibiotic usage for medical purposes may also have coincidently, contributed to the decline of the MS and indirectly to dental decay. This possibility led us to re-examine our data files on S. mutans (22-24), to find situations in which information on antibiotic usage had been obtained in order to determine whether a relationship between antibiotic usage and a decline in MS and caries could be demonstrated.

\section{Material and methods}

Coldwater. All children attending Grades 1 and 2 in the Coldwater Michigan school system (age 6 to 7 years) were given information concerning a prospective clinical study seeking to show a relationship between MS colonization and dental decay (8). A questionnaire on antibiotic history was distributed to the parents of the 411 children enrolled in the study and answers were received from 390 subjects, a $94 \%$ response rate. The antibiotic questions related to the parents recall as to whether their children used antibiotics on an average 1) 3 or more times per year; 2) once or twice per year; 3 ) less than once per year; or 4) never. The responses from these 390 subjects were tabulated and became the independent variable upon which a) the initial caries score in the primary teeth and b) the proportions of selected bacteria in plaque samples taken from the occlusal fissures were stratified.

All permanent and primary teeth were examined for dental decay by the same examiner. Caries was recorded by the decayed-filled surfaces (dfs) index in primary teeth. Caries was diagnosed on all coronal tooth surfaces only when softness or a definite break in the continuity of the enamel surface could be detected with an explorer. No bite-wing radiographs were taken.

Plaque samples for microbiological assay were taken from the occlusal surfaces of the lower first molars (2 samples per patient) with a No. 26-gauge sterile needle held with a hemostat. Each needle which contained from $10^{3}$ to $107 \mathrm{co-}$ lony-forming-units (CFU) of bacteria was dropped into a reduced transport fluid (RTF) (23) and transported back to the laboratory. After dispersion, serial dilution in RTF and plating, the plaque samples were incubated anaerobically under an atmosphere of $85 \%$ $\mathrm{N} 2,10 \% \mathrm{H}_{2}$ and $5 \% \mathrm{CO}_{2}$ within 3 to 5 $\mathrm{h}$ after their collection. The media used and the procedures for counting the colonies of MS, Streptococcus sanguis, lactobacilli, Veillonella and Actinomyces have been described previously (24). A ratio of the MS to $S$. sanguis was calculated for each plaque sample (23). In plaques where $S$. sanguis outnumbered the MS, the reciprocal value was calculated and reported as a negative number. The lower first molars were sampled as the colonization of their fissures within 6 months after their eruption was thought to reflect the "load" of MS that was present on the primary teeth.

Infants. The study population consisted of ten infants who were edentulous at the start of the observation period and who lived in households where at least two of the family members had levels of MS at or above $1 \times 10^{4} \mathrm{CFU}$ per $\mathrm{ml}$ of saliva. Bacterial samples of the edentulous infants were taken at approximately 14-day intervals by swabbing the oral mucosa overlying the mandibular and maxillary ridges with a sterile cottontipped applicator. This applicator was rubbed across the sterile surface of an MM10-10\% sucrose-bacitracin $(3 \mu \mathrm{g} /$ ml)-agar (MM10-SB agar) (23). As soon as 2 or more teeth had fully erupted in each infant the swabbing was discontinued in favor of sampling each tooth surface with unwaxed dental floss secured in a sterile floss holder. That portion of the floss containing the plaque was removed from the holder by cutting the floss with sterile scissors and placing it in the RTF.

All saliva (household members) and plaque samples were processed within one hour after their collection. They were sonified for $1000 \mathrm{watt} / \mathrm{s}$, serially diluted in RTF and plated on the MM10-SB agar and MSB agar plates (11), using a spiral plating technique (23). The sampling of the children was repeated at 2 to 3 week intervals over a 5 to 17 -month period of observation. Each infant was monitored for usage of antibiotics for medical purposes over this time interval. This information was obtained from the mother when contacted by telephone to schedule a sampling appointment or when questioned about the infants health at the time of sampling. These antibiotics were mainly oral penicillins prescribed for ear infections, "strep" throat, or bronchial infections.

\section{Results}

The survey of self-reported antibiotic usage in the Coldwater children showed that only $7 \%$ of the subjects had never received antibiotics; $49 \%$ received them less than one occasion per year; $32 \%$ received them one to two times per year and $12 \%$ received them on an average 3 or more times per year. It was not

Table 1. Mean percentage of selected plaque species in occlusal fissure plaque as a function of reported usage of antibiotics - Coldwater

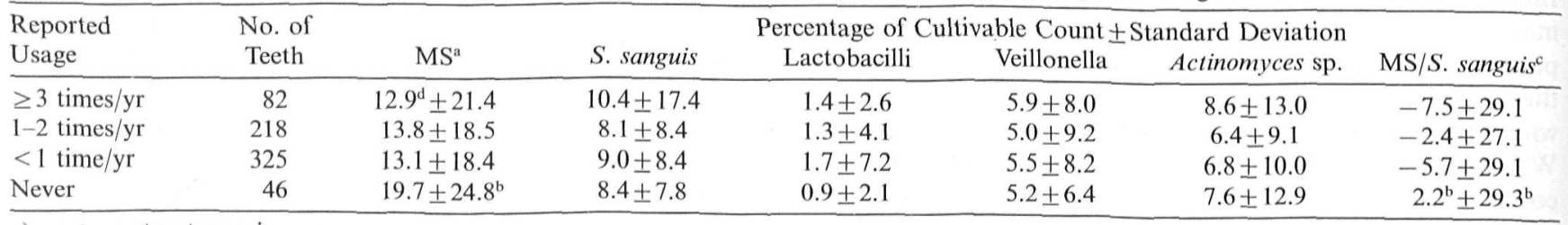
a) mutans streptococci.
b) value is significantly different; $\mathrm{p}=0.03$ Scheffe contrast of never $v$ all other categories.
c) negative values are the reciprocal of $\mathrm{MS} / S$. sanguis ratio, when $S$, sanguis is greater than the MS. 
possible to identify the types of antibiotics used.

The teeth in the Coldwater subjects who never received antibiotics had significantly higher proportions of MS in the fissure plaques of newly-erupting first molars than did the teeth in the subjects who received antibiotics $(\mathrm{p}=$ 0.03 , Scheffe contrast (Table 1). Within the antibiotic users there was no obvious effect of frequency of usage upon the percentage of MS. The reduction in MS was exceptional among the plaque flora that were monitored, as no antibiotic related changes in the proportions of S. sanguis, lactobacilli, Veillonella, or an unspeciated Actinomyces species was found (Table 1). When the MS to $S$. sanguis ratio was calculated (23), the MS outnumbered $S$. sanguis only in the children who had never received antibiotics (Table 1). In the other groups the MS to $S$. sanguis ratio was negative, indicating the relative dominance of $S$. sanguis in the fissure plaques.

The level of decay in the primary dentition was inversely related to the reported usage of antibiotics. Children who had never received antibiotics had significantly more decay than children who had received antibiotics 3 or more times per year (Table 2). Children who had received antibiotics 2 or less times per year had levels of decay that were intermediate to the "never" and " 3 or more" group. All antibiotic groups and the never group had comparable numbers of primary teeth (13.5 to 14.2), (Table 2), so that missing teeth did not seem to account for the observed differences.

Healthy infants living in households in which two or more family members had salivary MS levels higher than $10^{4}$ / $\mathrm{ml}$ were followed for periods ranging from 6 to 16 months after their teeth erupted. Only 2 children became detectably colonized by MS. One had never

Table 3. Relation between antibiotic therapy in infants and detection of mutans streptococci (MS) on primary teeth

\begin{tabular}{lccccc}
\hline Household & $\begin{array}{c}\text { MS } \\
\text { Detected }\end{array}$ & $\begin{array}{c}\text { Antibiotic } \\
\text { therapy }\end{array}$ & $\begin{array}{c}\text { Days on } \\
\text { antibiotic }\end{array}$ & $\begin{array}{c}\text { Month } \\
\text { dentate }\end{array}$ & $\begin{array}{c}\text { \% of dentate time } \\
\text { on antibiotics }\end{array}$ \\
\hline F 2 & yes (16) & no & 0 & 11 & 0.0 \\
F 12 & yes (8) & yes & 5 & 9 & 1.7 \\
F 5 & no (16) & no & 0 & 16 & 0.0 \\
F 4 & no (12) & yes & 13 & 14 & 3.0 \\
F 8 & no (6) & yes & 10 & 6 & 4.9 \\
F 11 & no (6) & yes & 13 & 7 & 5.0 \\
F 9 & no (5) & yes & 19 & 6 & 9.4 \\
F 7 & no (8) & yes & 84 & 10 & 29.1 \\
F 3 & no (12) & yes & 181 & 12 & 48.8 \\
F 10 & no (2) & yes & 90 & 6 & 64.1
\end{tabular}

a) number of teeth erupted at conclusion of study.

received antibiotics, whereas, the second had been on antibiotics for a single 5-day period (Table 3).MS were not detected on any teeth in the other 8 children even though sampling continued on a 2-3 week basis over a 6 to 16 month interval. Seven of these 8 children had received antibiotic therapy mainly with penicillin type medication for periods ranging from 10 to 181 days.

The number of days the teeth were exposed to antibiotics was divided by the number of days the teeth were in the mouth. The resultant values indicated that teeth not colonized by MS were exposed to antibiotics for periods ranging from $3 \%$ to $65 \%$ of their existence in the mouth (Table 3 ). The proportion of dentate time that the infants were reportedly on antibiotics was ranked according to whether or not their teeth became infected with MS. This ranking just missed statistical significance (Kruskal-Wallis, $\mathrm{p}=0.06$ ).

\section{Discussion}

The results described were obtained retrospectively from studies designed for other purposes, and are deficient in many respects, i.e., the type of antibiotics used, were the subjects, es-

Table 2. Mean number of decayed and filled primary teeth as a function of reported level of antibiotic use at baseline - Coldwater

\begin{tabular}{lcccccc}
\hline $\begin{array}{l}\text { Reported } \\
\text { Antibiotic } \\
\text { Use }\end{array}$ & $\begin{array}{c}\text { No. of } \\
\text { children }\end{array}$ & $\begin{array}{c}\text { Mean No. of } \\
\text { Primary Teeth }\end{array}$ & $\mathrm{dt}^{\mathrm{a}}$ & $\mathrm{ft}$ & $\mathrm{d}+\mathrm{ft}^{\mathrm{a}}$ & $\mathrm{p} \mathrm{value}^{\mathrm{b}}$ \\
\hline Never & 25 & $13.5 \pm 3.4^{\mathrm{c}}$ & 1.0 & 1.8 & 2.9 & 0.05 \\
$<1 /$ year & 192 & $14.0 \pm 2.8$ & 0.8 & 1.5 & 2.3 & 0.08 \\
1 to 2/year & 122 & $14.2 \pm 3.0$ & 0.7 & 1.7 & 2.4 & 0.06 \\
$\geq 3 /$ year & 51 & $14.2 \pm 3.1$ & 0.6 & 1.0 & 1.5 & \\
\hline
\end{tabular}

a) $\mathrm{d}=$ decayed primary teeth; $\mathrm{f}=$ filled teeth.

b) $\mathrm{p}$ value obtained for comparison of 3 or more per year group with each of the other groups, Scheffe contrast.

c) mean \pm standard deviation. pecially the infants, on antibiotics during sampling? Thus the findings should be interpreted with caution. Both the cross-sectional Coldwater study and the prospective study of infants suggest a relationship between frequency of antibiotic usage and MS levels on the teeth. The Coldwater study further suggested that increased antibiotic usage was associated with a reduced incidence of decay in the primary dentition.

The information on antibiotic usage in both studies was obtained by inquiry of the parents which could be considered as potentially unreliable, especially in the Coldwater study where longterm recall was involved. One might expect in the Coldwater study that the identification of those children who either never used antibiotics, or used them 3 or more times per year, to be the most reliable data obtained from the questionnaire, as the parents would be likely to recall this information accurately. It was the differences between these "never" and "frequent" antibiotic users that were statistically significant both in terms of proportions of the MS in fissure plaque (Table 1) and df scores (Table 2).

Maltz and Zickert (25) have shown that a single 10-day episode of penicillin significantly reduced the salivary levels of MS for several weeks, but had a minimal effect on $S$. sanguis and lactobacilli. A similar reduction in plaque MS proportions without a reduction in $S$. sanguis and lactobacilli was found in the antibiotic users relative to the non-antibiotic users in the Coldwater population. This bacteriological similarity between the 2 studies suggests that the questionnaire used in the present study may have reliably identified the nonantibiotic users.

An association between frequent antibiotic usage and a decrease in dental 
caries has been noted previously in patients with exceptional medical histories. Two retrospective epidemiologic studies showed that young rheumatic fever patients on a daily prophylactic penicillin regimen had significant percentage reductions in caries levels when compared to untreated controls $(14,20)$. Children with immunodeficiencies who were extensively, but not continually, treated with antibiotics had a mean DMF tooth score of 2.3, whereas immunodeficient children who averaged only 5 days on atibiotics per year had a DMF tooth score of 5.4 (28). A study of the caries experience of children with tetracycline discolored teeth showed a modest, but significantly lower DMFS scores for the complete dentition, deciduous molars and first permanent molars in comparison with paired control dentitions in children without any discoloration (5).

These findings suggest that very frequent usage of antibiotics in medically compromised children can reduce the incidence of dental decay. This can be envisioned as due to the more or less continued suppression of the MS in the plaque. However, in medically healthy children such as our Coldwater subjects, in order for the medical usage of antibiotics to be coincidentally lowering dental decay, it is necessary to posit that the temporary suppression of MS in the plaques occurs during a stage or a period in the tooth maturation, in which the tooth is most vulnerable to a cariogenic attach by the MS. This would be the period shortly after tooth eruption and would be dependent upon the MS being among the initial colonizers of the fissure depths (22).

If during the period of tooth eruption, the child is receiving or has just received antibiotics that have activity against the MS, there is the possibility that the salivary levels of the MS will be so low that the fissure will become colonized by bacteria other than the MS (29). There is data that shows if the MS colonization of the dentition is delayed or does not occur, then the caries experience in the primary dentition is quite low or absent, whereas if colonization occurs shortly after eruption, decay is common. Thus, when the MS were not detected in 2-year-old children, these children were caries free at age 4, whereas children with detectable MS at age 2 had a df score of over 10 at age 4 (1). In another investigation, only 1 of 36 children without detectable MS coloniz- ation in their first 4 years of life had decay, whereas $75 \%$ of the children infected by age 15 months had decay by age 4 (19). If primary incisors become colonized with MS shortly after eruption, they soon become carious (27).

These findings, combined with the results from our infant study, provide a connection between antibiotic usage and reduced colonization of the MS in the primary dentition. In 7 of these infants frequent usage of antibiotics and absence of MS were apparently linked events. If the antibiotic usage in these 10 infants is representative of its usage by other children, such as those living in Coldwater, then the antibiotic associated reduction in decay and MS proportions noticed in the Coldwater children could reflect absent or delayed colonization by the MS when the teeth erupted and were most prone to a cariogenic challenge. This colonization-delayed, infection-prevented model could then account for some of the decline in dental decay that has been noted in many countries. However, the actual demonstration of this effect would require a prospective study where types and duration of antibiotic usage could be correlated with data on MS colonization and levels, and actual caries experience of the children.

\section{Acknowledgements}

This research was supported by grants DE02731 and DE04504.

\section{References}

1. Alaluusua S, Renkonen OV. Streptococcus mutans establishment and dental caries experience in children from 2- to 4 years-old. Scand J Dent Res 1983: 91: 453-457.

2. Axelsson P, Lindhe J. Effect of oral hygiene instruction and professional toothcleaning on caries and gingivitis in schoolchildren. Community Dent Oral Epidemiol 1981: 9: 251-255

3. Bohannan HM. The impact of decreasing caries prevalence: Implications for dental education. J Dent Res 1982: 61: 1369-1377.

4. Bowden GHW, Odlum O, Nolette N, Hamilton IR. Microbial populations growing in the presence of fluoride at low $\mathrm{pH}$ isolated from dental plaque of children living in an area with fluoridated water. Infect Immun 1982: 36: 247-254.

5. Brearly LJ, Porteus JR. Characteristics and experience of tetracycline-affected dentitions. J Dent Res 1973: 52: 508-516.
6. Brunelle JA, Carlos JP. Changes in the prevalence of dental caries in U.S. school children, 1961-1980. J Dent Res 1982: 61 (spec. iss.): 1346-1351.

7. Burt BA. The future of the caries decline. J Public Health Dent 1985: 45: 261-269.

8. Burt BA, Loesche WJ, Eklund SA. Stability of selected plaque species and their relationship to caries in a child population over 2 years. Caries Res 1985: 19: 193-200.

9. Fitzgerald RJ, Keyes PH. Demonstration of the etiologic role of streptococci in experimental caries in the hamster. J Am Dent Assoc 1960: 61: 9-19.

10. Glass RL. The first international conference on the declining prevalence of dental caries. J Dent Res 1982: 61: 1301-1383.

11. Gold OC, Jordan HV, Van Houte J. A selective medium for Streptococcus mutans. Arch Oral Biol 1973: 18: 1356-1364.

12. Gordis L. The virtual disappearance of rheumatic fever in the United States: lesions in the rise and fall of disease. Circulation. 1985: 72: 1155-1162.

13. Granath LE, Martinsson T, Matsson L, Nilsson G, Schroder U, Soderholm B. Intra-individual effect of daily supervised flossing on caries in school children. Comm Dent Oral Epidemiol 1979: 7: 147-150.

14. Handelman SL, Hawes RR. The effect of long-term antibiotic therapy on antibiotic resistance of the salivary flora. $\mathrm{J}$ Oral Ther Pharm 1964: 1: 23-44.

15. Horowitz AM, Suomi JD, Peterson JK, Lyman AB. Effects of supervised daily dental plaque removal by children. II. 24 months' results. J Public Health Dent 1977: 37: 180-188.

16. Keyes PH. The infectious and transmissible nature of experimental dental caries. Findings and implications. Arch Oral Biol 1960: 1: 304-320.

17. Klock B, Krasse B. Caries status and microbial conditions in children in 1973 and 1984. Scand J Dent Res 1987: 95: 13-17.

18. Koch $\mathrm{G}$. Evidence for declining caries prevalence in Sweden. J Dent Res 1982: 61: $1340-1345$.

19. Kohler B, Andreen I, Jonsson B. The effect of caries-preventive measures in mothers on dental caries and the oral presence of the bacteria Streptococcus mutans and lactobacilli in their children. Arch Oral Biol 1984: 29: 879-883.

20. Littleton NW, White CF. Dental findings from a preliminary study of children receiving antibiotic therapy. J Amer Dent Assoc 1964: 68: 520-525.

21. Loesche WJ. Role of Streptococcus mutans in human dental decay. Microbiol Rev. 1986a: 50: 353-380.

22. Loesche WJ. Decline in Streptococcus mutans-associated caries secondary to medical usage of antibiotics. In: Molecular Microbiology and immunobiology of 
Streptococcus mutans. Hamada et al., eds. Amsterdam: Elsevier 1986b. pp.371-380.

23. Loesche WJ, Straffon LH. Longitudinal investigation of the role of Streptococcus mutans in human fissure decay. Infect Immun 1979: 26: 498-507.

24. Loesche WJ, Eklund S, Earnest R, Burt B. Longitudinal investigation of bacteriology of human fissure decay: epidemiological studies in molars shortly after eruption. Infect Immun 1984: 46: 765-772.

25. Maltz M, Zickert I. Effect of penicillin on Streptococcus mutans, Streptococcus sanguis, and lactobacilli in hamsters and in man. Scand J Dent Res 1982: 90: 193-199.

26. Massell BF, Chute CG, Walker AM, Kurland GS. Penicillin and the marked decrease in morbidity and mortality from rheumatic fever in the United States. N Engl J Med 1988: 318: 280-286.

27. Masuda N, Tsutsumi N, Sobue S, Hamada S. Longitudinal survey of the distribution of various serotypes of Streptococcus mutans in infants. J Clin Microbiol 1979: 10: 497-502.

28. Robertson PB, Wright TE III, Mackler
BF, Lenertz DM, Levy BM. Periodontal status of patients with abnormalities of the immune system. J Periodont Res 1978: 13: 37-45.

29. Svanberg M, Loesche WJ. Salivary concentration of Streptococcus mutans and Streptococcus sanguis and the colonization of artificial fissures in humans by these organisms. Arch Oral Biol 1977: 22: 441-447.

30. Van Houte J, Aasenden R, Peebles TC. Oral colonization of Streptococcus mutans in human subjects with low caries experience given fluoride supplements from birth. Arch Oral Biol 1978: 23: 361-366. 
This document is a scanned copy of a printed document. No warranty is given about the accuracy of the copy. Users should refer to the original published version of the material. 